\title{
On the Spectrum of Two-Point Boundary Value Problems for the Dirac Operator
}

\author{
A. S. Makin ${ }^{1 *}$ \\ ${ }^{1}$ MIREA-Russian Technological University, Moscow, 119454 Russia \\ e-mail: *alexmakin@yandex.ru
}

Received July 20, 2020; revised July 20, 2020; accepted June 8, 2021

\begin{abstract}
We consider the spectral problem for a Dirac operator with arbitrary two-point boundary conditions and an arbitrary complex-valued integrable potential. The existence of nontrivial boundary value problems of this type with an unbounded growth of the multiplicity of eigenvalues is established.
\end{abstract}

DOI: $10.1134 /$ S0012266121080036

\section{INTRODUCTION}

In the present paper, we study the Dirac system

$$
B \mathbf{y}^{\prime}+V \mathbf{y}=\lambda \mathbf{y}
$$

where $\mathbf{y}=\operatorname{col}\left(y_{1}(x), y_{2}(x)\right), \lambda \in \mathbb{C}$ is the spectral parameter,

$$
B=\left(\begin{array}{cc}
0 & 1 \\
-1 & 0
\end{array}\right), \quad V(x)=\left(\begin{array}{cc}
p(x) & q(x) \\
q(x) & -p(x)
\end{array}\right),
$$

and the functions $p, q \in L_{1}(0, \pi)$ are complex-valued, with the two-point boundary conditions

$$
U(\mathbf{y}) \equiv C \mathbf{y}(0)+D \mathbf{y}(\pi)=0
$$

where

$$
C=\left(\begin{array}{ll}
a_{11} & a_{12} \\
a_{21} & a_{22}
\end{array}\right), \quad D=\left(\begin{array}{ll}
a_{13} & a_{14} \\
a_{23} & a_{24}
\end{array}\right),
$$

the coefficients $a_{i j}$ can be any complex numbers, and the rows of the matrix

$$
A=(C D)=\left(\begin{array}{llll}
a_{11} & a_{12} & a_{13} & a_{14} \\
a_{21} & a_{22} & a_{23} & a_{24}
\end{array}\right)
$$

are linearly independent.

We denote by $\|f\|=\left(\left|f_{1}\right|^{2}+\left|f_{2}\right|^{2}\right)^{1 / 2}$ the norm of an arbitrary vector $f=\operatorname{col}\left(f_{1}, f_{2}\right) \in \mathbb{C}^{2}$ and set $\langle f, g\rangle=f_{1} g_{1}+f_{2} g_{2}$. We denote the norm of an arbitrary $2 \times 2$ matrix $W$ by $\|W\|=\sup _{\|f\|=1}\|W f\|$. Let $L_{2,2}(a, b)$ be the space of two-dimensional vector functions $f(t)=\operatorname{col}\left(f_{1}(t), f_{2}(t)\right)$ with the norm $\|f\|_{L_{2,2}(a, b)}=\left(\int_{a}^{b}\|f(t)\| d t\right)^{1 / 2}$, and let $L_{2,2}^{2,2}(a, b)$ be the space of $2 \times 2$ matrix functions $W(t)$ with the norm $\|W\|_{L_{2,2}^{2,2}(a, b)}=\left(\int_{a}^{b}\|W(t)\| d t\right)^{1 / 2}$. We treat the operator $\mathbb{L} \mathbf{y}=B \mathbf{y}^{\prime}+V \mathbf{y}$ as a linear operator in the space $L_{2,2}(0, \pi)$ with domain $D(\mathbb{L})=\left\{\mathbf{y} \in W_{1}^{1}[0, \pi]: \mathbb{L} \mathbf{y} \in L_{2,2}(0, \pi), U_{j}(\mathbf{y})=0\right.$ $(j=1,2)\}$.

Let

$$
E(x, \lambda)=\left(\begin{array}{cc}
c_{1}(x, \lambda) & -s_{2}(x, \lambda) \\
s_{1}(x, \lambda) & c_{2}(x, \lambda)
\end{array}\right)
$$


be the fundamental matrix of Eq. (1) with the boundary condition $E(0, \lambda)=I$, where $I$ is the identity matrix, and let $E_{0}(x, \lambda)$ be the fundamental matrix of the unperturbed equation $B \mathbf{y}^{\prime}=\lambda \mathbf{y}$ with the boundary condition $E_{0}(0, \lambda)=I$. It is obvious that

$$
E_{0}(x, \lambda)=\left(\begin{array}{cc}
\cos (\lambda x) & -\sin (\lambda x) \\
\sin (\lambda x) & \cos (\lambda x)
\end{array}\right)
$$

It is well known that the entries of the matrix $E(x, \lambda)$ are related by

$$
c_{1}(x, \lambda) c_{2}(x, \lambda)+s_{1}(x, \lambda) s_{2}(x, \lambda)=1
$$

for any $x$ and $\lambda$. Let $J_{i j}$ be the determinant formed by the $i$ th and $j$ th columns of $A$. Set $J_{0}=J_{12}+J_{34}, J_{1}=J_{14}-J_{23}$, and $J_{2}=J_{13}+J_{24}$.

It was shown in [1] by the transformation operator method that the characteristic determinant $\Delta(\lambda)$ of problem (1), (2), which is equal to

$$
\Delta(\lambda)=J_{12}+J_{34}+J_{14} c_{2}(\pi, \lambda)-J_{23} c_{1}(\pi, \lambda)-J_{13} s_{2}(\pi, \lambda)-J_{24} s_{1}(\pi, \lambda),
$$

can be reduced to the form

$$
\Delta(\lambda)=\Delta_{0}(\lambda)+\int_{0}^{\pi} r_{1}(t) e^{-i \lambda t} d t+\int_{0}^{\pi} r_{2}(t) e^{i \lambda t} d t=\Delta_{0}(\lambda)+R(\lambda),
$$

where the function

$$
\begin{aligned}
\Delta_{0}(\lambda) & =J_{0}+J_{1} \cos (\pi \lambda)-J_{2} \sin (\pi \lambda) \\
& =J_{12}+J_{34}+\frac{1}{2}\left(e^{i \pi \lambda}\left(J_{1}+i J_{2}\right)+e^{-i \pi \lambda}\left(J_{1}-i J_{2}\right)\right)=J_{0}+C_{1} e^{i \pi \lambda}+C_{2} e^{-i \pi \lambda},
\end{aligned}
$$

$C_{1}=\left(J_{1}+i J_{2}\right) / 2, C_{2}=\left(J_{1}-i J_{2}\right) / 2$, is the characteristic determinant of the unperturbed problem

$$
B \mathbf{y}^{\prime}=\lambda \mathbf{y}, \quad U(\mathbf{y})=0
$$

and the functions $r_{j}$ belong to the space $L_{1}(0, \pi), j=1,2$. If $p, q \in L_{2}(0, \pi)$ (for short, we write $\left.V \in L_{2}(0, \pi)\right)$, then $r_{j} \in L_{2}(0, \pi)$. It follows that the function $\Delta(\lambda)$ is an entire function of exponential type; therefore, we only have the following possibilities for the operator $\mathbb{L}$ of problem (1), (2):

1. The spectrum is empty.

2. The spectrum is a finite nonempty set.

3. The spectrum is a countable set without finite limit points.

4. The spectrum fills the entire complex plane.

Relations (5) and (6) imply that case 1 is realized for problem (7), for example, with the boundary conditions defined by the matrix

$$
A=\left(\begin{array}{cccc}
1 & i & -1 & i \\
1 & -i & 1 & i
\end{array}\right)
$$

and case 4 , with the boundary conditions defined by the matrix

$$
A=\left(\begin{array}{cccc}
1 & -i & 0 & 0 \\
0 & 0 & i & 1
\end{array}\right)
$$

Let us prove that case 2 is impossible. Let the equation

$$
\Delta(\lambda)=0
$$


have finitely many roots $\lambda_{k}, k=1, \ldots, n$. If $C_{1} C_{2} \neq 0$, then conditions (2) are regular and problem (1), (2) has a countable set of eigenvalues; therefore, $C_{1} C_{2}=0$. Set $P(\lambda)=\prod_{k=1}^{n}\left(\lambda-\lambda_{k}\right)$. By [2],

$$
\Delta(\lambda)=P(\lambda) e^{a \lambda+b},
$$

where $a$ and $b$ are some constants. Assume, for example, that $C_{2}=0$. Setting $\lambda=-i y$ in relation (5), where $y>0$, we obtain

$$
J_{0}+C_{1} e^{\pi y}+R(-i y)=P(-i y) e^{-i a y+b}
$$

which implies that

$$
J_{0} e^{-\pi y}+C_{1}+e^{-\pi y} R(-i y)=P(-i y) e^{b-i \operatorname{Re} a y} e^{(\operatorname{Im} a-\pi) y} .
$$

According to $\left[3\right.$, p. 36], the expression on the left-hand side in relation (8) tends to $C_{1}$ as $y \rightarrow \infty$. If $\operatorname{Im} a-\pi \geq 0$, then the expression on the right-hand side in relation (8) tends to infinity in absolute value, and if $\operatorname{Im} a-\pi<0$, then it tends to zero. It follows that $C_{1}=0$. If $C_{1}=C_{2}=0$, then

$$
R(\lambda)=P(\lambda) e^{a \lambda+b}
$$

Obviously, the left-hand side of relation (9) is bounded on the real axis, while the right-hand side is not; that is, we arrive at a contradiction.

Definition. We say that problem (1), (2) has the classical spectral asymptotics if its spectrum is a countable set and the multiplicities of the eigenvalues are uniformly bounded.

The present paper is aimed at constructing problems (1), (2) for which case 3 is realized and the multiplicities of the eigenvalues grow unboundedly, i.e., problems with nonclassical spectral asymptotics.

\section{MAIN RESULTS}

Set $c_{j}(\lambda)=c_{j}(\pi, \lambda)$ and $s_{j}(\lambda)=s_{j}(\pi, \lambda), j=1,2$. In addition, let $P W_{\sigma}$ be the class of entire functions $f(z)$ of the exponential type $\leq \sigma$ such that $\|f\|_{L_{2}(R)}<\infty$. It is well known [4] that the functions $c_{j}(\lambda)$ and $s_{j}(\lambda)$ admit the representation

$$
c_{j}(\lambda)=\cos (\pi \lambda)+g_{j}(\lambda), \quad s_{j}(\lambda)=\sin (\pi \lambda)+h_{j}(\lambda),
$$

where $g_{j}, h_{j} \in P W_{\pi}, j=1,2$.

Lemma 1 [5]. The functions $u(\lambda)$ and $v(\lambda)$ admit the representations

$$
u(\lambda)=\sin (\pi \lambda)+h(\lambda), \quad v(\lambda)=\cos (\pi \lambda)+g(\lambda),
$$

where $h, g \in P W_{\pi}$, if and only if

$$
u(\lambda)=-\pi\left(\lambda_{0}-\lambda\right) \prod_{\substack{n=-\infty \\ n \neq 0}}^{\infty} \frac{\lambda_{n}-\lambda}{n},
$$

where $\lambda_{n}=n+\varepsilon_{n}$ and $\left\{\varepsilon_{n}\right\} \in l_{2}$, and

$$
v(\lambda)=\prod_{n=-\infty}^{\infty} \frac{\lambda_{n}-\lambda}{n-1 / 2},
$$

where $\lambda_{n}=n-1 / 2+\kappa_{n}$ and $\left\{\kappa_{n}\right\} \in l_{2}$.

Consider the Dirac system with the boundary conditions defined by the matrix

$$
A=\left(\begin{array}{llll}
1 & 0 & 0 & 1 \\
0 & 1 & 1 & 0
\end{array}\right)
$$

DIFFERENTIAL EQUATIONS $\quad$ Vol. $57 \quad$ No. $8 \quad 2021$ 
We will assume that $V \in L_{2}(0, \pi)$. It follows from the representation (4) that the characteristic determinant $\Delta(\lambda)$ of problem (1), (2) with matrix $A$ defined in (10) can be reduced to the form

$$
\Delta(\lambda)=s_{1}(\lambda)-s_{2}(\lambda)=\int_{-\pi}^{\pi} r(t) e^{i \lambda t} d t=f(\lambda)
$$

where $r \in L_{2}(0, \pi)$, and $f \in P W_{\pi}$. The converse statement holds true as well.

Theorem. For each function $f \in P W_{\pi}$, there exists a potential $V \in L_{2}(0, \pi)$ such that the characteristic determinant $\Delta(\lambda)$ of problem (1), (2) with the matrix $A$ defined by relation (10) and the potential $V(x)$ is identically equal to $f(\lambda)$.

Proof. Let $f(\lambda)$ be an arbitrary function in the class $P W_{\pi}$. It follows from the Paley-Wiener theorem and [3, p. 36] that

$$
\lim _{|\lambda| \rightarrow \infty} e^{-\pi|\operatorname{Im} \lambda|} f(\lambda)=0 ;
$$

consequently, there exists a positive integer $N_{0}$ so large that $|f(\lambda)|<1 / 100$ if $\operatorname{Im} \lambda=0$ and $|\operatorname{Re} \lambda| \geq N_{0}$.

Let $\left\{\lambda_{n}\right\}, n \in \mathbb{Z}$, be a strictly monotone increasing sequence of real numbers such that $N_{0}<\lambda_{n}<N_{0}+1 / 100$ if $1 \leq n \leq N_{0}, \lambda_{n}=n-1 / 2$ if $n>N_{0}$, and $\lambda_{n}=-\lambda_{-n+1}$ for any $n$. Set

$$
c(\lambda)=\prod_{n=-\infty}^{\infty} \frac{\lambda_{n}-\lambda}{n-1 / 2} .
$$

Lemma 1 implies the relation

$$
c(\lambda)=\cos (\pi \lambda)+g(\lambda),
$$

where $g \in P W_{\pi}$. It follows from the Paley-Wiener theorem and [3, p. 36] that

$$
\lim _{|\lambda| \rightarrow \infty} e^{-\pi|\operatorname{Im} \lambda|} g(\lambda)=0 ;
$$

therefore,

$$
|c(\lambda)| \geq c_{0} e^{\pi|\operatorname{Im} \lambda|}
$$

$\left(c_{0}=\right.$ const $\left.>0\right)$ for $|\operatorname{Im} \lambda| \geq M$, where $M$ is a sufficiently large number.

Differentiating relation (12), we obtain

$$
\dot{c}(\lambda)=-\pi \sin (\pi \lambda)+\dot{g}(\lambda) .
$$

Since the function $\dot{g}$ belongs to the class $P W_{\pi}$, we have, according to [6],

$$
\dot{c}\left(\lambda_{n}\right)=-\pi \sin \left(\pi \lambda_{n}\right)+\tau_{n}
$$

where

$$
\sum_{n=-\infty}^{\infty}\left|\tau_{n}\right|^{2}<\infty
$$

Based on this, by the definition of the numbers $\lambda_{n}$, we obtain

$$
\dot{c}\left(\lambda_{n}\right)=\pi(-1)^{n}+\rho_{n},
$$

where

$$
\sum_{n=-\infty}^{\infty}\left|\rho_{n}\right|^{2}<\infty
$$


Consequently, for all even $n$ sufficiently large in modulus one has the inequality $\dot{c}\left(\lambda_{n}\right)>0$. One can readily see that the inequality $\dot{c}\left(\lambda_{n}\right) \dot{c}\left(\lambda_{n+1}\right)<0$ holds for all $n \in \mathbb{Z}$. It follows that

$$
(-1)^{n} \dot{c}\left(\lambda_{n}\right)>0
$$

for all $n \in \mathbb{Z}$. Note that (15) implies the relation

$$
\frac{1}{\dot{c}\left(\lambda_{n}\right)}=\frac{(-1)^{n}}{\pi}+\sigma_{n}
$$

where

$$
\sum_{n=-\infty}^{\infty}\left|\sigma_{n}\right|^{2}<\infty
$$

Consider the quadratic equation

$$
w^{2}+f\left(\lambda_{n}\right) w-1=0 .
$$

It has the roots

$$
s_{n}^{ \pm}=\frac{-f\left(\lambda_{n}\right) \pm \sqrt{f^{2}\left(\lambda_{n}\right)+4}}{2} .
$$

By $\Gamma(z, r)$ we denote the disk of radius $r$ centered at point $z$. One can readily see that all numbers $s_{n}^{+}$ lie inside the disk $\Gamma(1,1 / 10)$ and all numbers $s_{n}^{-}$lie inside the disk $\Gamma(-1,1 / 10)$. Let $s_{n}=s_{n}^{+}$if $n$ is odd and $s_{n}=s_{n}^{-}$if $n$ is even. Since [6] $\left\{f\left(\lambda_{n}\right)\right\} \in l_{2}$, it follows from the definition of the numbers $s_{n}$ that

$$
s_{n}=(-1)^{n+1}+\vartheta_{n},
$$

where $\left\{\vartheta_{n}\right\} \in l_{2}$. It also follows from the definition of the numbers $s_{n}$ and inequality (16) that all numbers $z_{n}=s_{n} / \dot{c}\left(\lambda_{n}\right)$ lie strictly to the left of the imaginary axis, while (17) and (19) imply the relation

$$
z_{n}=-\frac{1}{\pi}+\rho_{n},
$$

where $\left\{\rho_{n}\right\} \in l_{2}$. Let $\beta_{n}=s_{n}-\sin \left(\pi \lambda_{n}\right)$; then $\left\{\beta_{n}\right\} \in l_{2}$ in view of (19). Set

$$
h(\lambda)=c(\lambda) \sum_{n=-\infty}^{\infty} \frac{\beta_{n}}{\dot{c}\left(\lambda_{n}\right)\left(\lambda-\lambda_{n}\right)} .
$$

According to [7, p. 120], the function $h$ belongs to the class $P W_{\pi}$, and $h\left(\lambda_{n}\right)=\beta_{n}$. Set $s(\lambda)=\sin (\pi \lambda)+h(\lambda)$; then $s\left(\lambda_{n}\right)=s_{n} \neq 0$, and consequently, the functions $s(\lambda)$ and $c(\lambda)$ do not have common roots.

Set

$$
Y_{0}(x, \lambda)=\left(\begin{array}{c}
\cos (\lambda x) \\
\sin (\lambda x)
\end{array}\right)
$$

In the subsequent exposition, we need the following elementary assertion.

Lemma 2. If function systems $\left\{\varphi_{n}\right\}$ and $\left\{\psi_{n}\right\}$ are complete in $L_{2}(a, b)(n \in \mathbb{N})$, then the system of vectors

$$
\Psi_{n, n}=\left(\begin{array}{c}
\left\{\varphi_{n}\right\} \\
\left\{\psi_{n}\right\}
\end{array}\right) \cup\left(\begin{array}{c}
\left\{\varphi_{n}\right\} \\
\left\{-\psi_{n}\right\}
\end{array}\right)
$$

is complete in $L_{2,2}(a, b)$.

Proof. Assume that there exists a vector $f(x)=\operatorname{col}\left(f_{1}(x), f_{2}(x)\right) \neq 0$ such that

$$
\int_{a}^{b}\left(\varphi_{n}(x) \overline{f_{1}(x)}+\psi_{n}(x) \overline{f_{2}(x)}\right) d x=0, \quad \int_{a}^{b}\left(\varphi_{n}(x) \overline{f_{1}(x)}-\psi_{n}(x) \overline{f_{2}(x)}\right) d x=0
$$

DIFFERENTIAL EQUATIONS Vol. 57 No. $8 \quad 2021$ 
for all $n \in \mathbb{N}$. Then

$$
\int_{a}^{b} \varphi_{n}(x) \overline{f_{1}(x)} d x=0, \quad \int_{a}^{b} \psi_{n}(x) \overline{f_{2}(x)} d x=0 ;
$$

consequently, $f_{1}(x) \equiv f_{2}(x) \equiv 0$. The proof of the lemma is complete.

It follows from [8] that the function systems $\left\{\cos \left(\lambda_{n} x\right)\right\}$ and $\left\{\sin \left(\lambda_{n} x\right)\right\}(n \in \mathbb{N})$ are complete in $L_{2}(0, \pi)$. Based on this, it follows from the definition of the numbers $\lambda_{n}$ and Lemma 2 that the system of vectors

$$
Y_{0}\left(x, \lambda_{n}\right)=\left(\begin{array}{c}
\cos \left(\lambda_{n} x\right) \\
\sin \left(\lambda_{n} x\right)
\end{array}\right)
$$

$(n \in \mathbb{Z})$ is complete in $L_{2,2}(0, \pi)$. Set

$$
F(x, t)=-\sum_{n=-\infty}^{\infty}\left(\frac{s_{n}}{\dot{c}\left(\lambda_{n}\right)}\left(Y_{0}\left(x, \lambda_{n}\right) Y_{0}^{\mathrm{T}}\left(t, \lambda_{n}\right)\right)+\frac{1}{\pi} Y_{0}(x, n-1 / 2) Y_{0}^{\mathrm{T}}(t, n-1 / 2)\right) .
$$

It follows from [4] that

$$
\|F(\cdot, x)\|_{L_{2,2}^{2,2}(0, \pi)}+\|F(x, \cdot)\|_{L_{2,2}^{2,2}(0, \pi)}<C,
$$

where $C$ is a constant independent of $x$. Let us prove that for each $x \in[0, \pi]$ the homogeneous equation

$$
f^{\mathrm{T}}(t)+\int_{0}^{x} f^{\mathrm{T}}(s) F(s, t) d s=0,
$$

where $f(t)=\operatorname{col}\left(f_{1}(t), f_{2}(t)\right), f \in L_{2,2}(0, x), f(t)=0$ for $x<t \leq \pi$, has only the trivial solution. Multiplying Eq. (21) by $\overline{f^{\mathrm{T}}(t)}$ and integrating the resulting equation over the segment $[0, x]$, we obtain

$$
\|f\|_{L_{2,2}(0, x)}^{2}+\int_{0}^{x}\left\langle\int_{0}^{x} f^{\mathrm{T}}(s) F(s, t) d s, \overline{f^{\mathrm{T}}(t)}\right\rangle d t=0 .
$$

Taking into account definition (20), by simple calculations we find that

$$
\begin{aligned}
& f^{\mathrm{T}}(s) F(s, t) \\
& =-\left\{\sum _ { n = - \infty } ^ { \infty } \left\{z _ { n } \left[f_{1}(s) \cos \left(\lambda_{n} s\right) \cos \left(\lambda_{n} t\right)+f_{2}(s) \sin \left(\lambda_{n} s\right) \cos \left(\lambda_{n} t\right),\right.\right.\right. \\
& \left.\quad f_{1}(s) \cos \left(\lambda_{n} s\right) \sin \left(\lambda_{n} t\right)+f_{2}(s) \sin \left(\lambda_{n} s\right) \sin \left(\lambda_{n} t\right)\right] \\
& +\frac{1}{\pi}\left[f_{1}(s) \cos ((n-1 / 2) s) \cos ((n-1 / 2) t)+f_{2}(s) \sin ((n-1 / 2) s) \cos ((n-1 / 2) t),\right. \\
& \left.\left.\left.\quad f_{1}(s) \cos ((n-1 / 2) s) \sin ((n-1 / 2) t)+f_{2}(s) \sin ((n-1 / 2) s) \sin ((n-1 / 2) t)\right]\right\}\right\} \\
& +\frac{1}{\pi}\left[f_{1}(s) \cos ((n-1 / 2) s) \cos ((n-1 / 2) t)+f_{2}(s) \sin ((n-1 / 2) s) \cos ((n-1 / 2) t)\right], \\
& \left.\left.+\frac{1}{\pi}\left[f_{1}(s) \cos (((n-1 / 2) s)) \sin ((n-1 / 2) t) f_{2}(s) \sin ((n-1 / 2) s) \sin ((n-1 / 2) t)\right]\right\}\right\}
\end{aligned}
$$


which implies that

$$
\begin{aligned}
& \int_{0}^{x}\left\langle\int_{0}^{x} f^{\mathrm{T}}(s) F(s, t) d s, \overline{f^{\mathrm{T}}(t)}\right\rangle d t \\
& =-\left\{\sum _ { n = - \infty } ^ { \infty } \int _ { 0 } ^ { x } \left(\int _ { 0 } ^ { x } \left\{z_{n}\left[f_{1}(s) \cos \left(\lambda_{n} s\right) \cos \left(\lambda_{n} t\right)+f_{2}(s) \sin \left(\lambda_{n} s\right) \cos \left(\lambda_{n} t\right)\right]\right.\right.\right. \\
& +\frac{1}{\pi}\left[f_{1}(s) \cos ((n-1 / 2) s) \cos ((n-1 / 2) t)\right. \\
& \left.\left.\left.+f_{2}(s) \sin ((n-1 / 2) s) \cos ((n-1 / 2) t)\right]\right\} d s\right) \overline{f_{1}(t)} d t \\
& +\sum_{n=-\infty}^{\infty} \int_{0}^{x}\left(\int _ { 0 } ^ { x } \left\{z_{n}\left[f_{1}(s) \cos \left(\lambda_{n} s\right) \sin \left(\lambda_{n} t\right)+f_{2}(s) \sin \left(\lambda_{n} s\right) \sin \left(\lambda_{n} t\right)\right]\right.\right. \\
& +\frac{1}{\pi}\left[f_{1}(s) \cos ((n-1 / 2) s) \sin ((n-1 / 2) t)\right. \\
& \left.\left.\left.\left.+f_{2}(s) \sin ((n-1 / 2) s) \sin ((n-1 / 2) t)\right]\right\} d s\right) \overline{f_{2}(t)} d t\right\} \\
& =-\left\{\sum _ { n = - \infty } ^ { \infty } \left(\int_{0}^{x} z_{n}\left[f_{1}(s) \cos \left(\lambda_{n} s\right)+f_{2}(s) \sin \left(\lambda_{n} s\right)\right] d s \int_{0}^{x} \cos \left(\lambda_{n} t\right) \overline{f_{1}(t)} d t\right.\right. \\
& \left.+\frac{1}{\pi} \int_{0}^{x}\left[f_{1}(s) \cos ((n-1 / 2) s)+f_{2}(s) \sin ((n-1 / 2) s)\right] d s \int_{0}^{x} \cos ((n-1 / 2) t) \overline{f_{1}(t)} d t\right) \\
& +\sum_{n=-\infty}^{\infty}\left(\int_{0}^{x} z_{n}\left[f_{1}(s) \cos \left(\lambda_{n} s\right)+f_{2}(s) \sin \left(\lambda_{n} s\right)\right] d s \int_{0}^{x} \sin \left(\lambda_{n} t\right) \overline{f_{2}(t)} d t\right. \\
& \left.\left.+\frac{1}{\pi} \int_{0}^{x}\left[f_{1}(s) \cos ((n-1 / 2) s)+f_{2}(s) \sin ((n-1 / 2) s)\right] d s \int_{0}^{x} \sin ((n-1 / 2) t) \overline{f_{2}(t)} d t\right)\right\} \\
& =-\left\{\sum _ { n = - \infty } ^ { \infty } \left(\int_{0}^{x} z_{n}\left[f_{1}(s) \cos \left(\lambda_{n} s\right)+f_{2}(s) \sin \left(\lambda_{n} s\right)\right] d s \int_{0}^{x} \cos \left(\lambda_{n} t\right) \overline{f_{1}(t)} d t\right.\right. \\
& \left.+\int_{0}^{x}\left[f_{1}(s) \cos \left(\lambda_{n} s\right)+f_{2}(s) \sin \left(\lambda_{n} s\right)\right] d s \int_{0}^{x} \sin \left(\lambda_{n} t\right) \overline{f_{2}(t)} d t\right) \\
& +\frac{1}{\pi} \sum_{n=-\infty}^{\infty}\left(\int_{0}^{x}\left[f_{1}(s) \cos ((n-1 / 2) s)+f_{2}(s) \sin ((n-1 / 2) s)\right] d s\right) \int_{0}^{x} \cos ((n-1 / 2) t) \overline{f_{1}(t)} d t \\
& \left.\left.+\int_{0}^{x}\left[f_{1}(s) \cos ((n-1 / 2) s)+f_{2}(s) \sin ((n-1 / 2) s)\right] d s \int_{0}^{x} \sin ((n-1 / 2) t) \overline{f_{2}(t)} d t\right)\right\}
\end{aligned}
$$

DIFFERENTIAL EQUATIONS $\quad$ Vol. $57 \quad$ No. $8 \quad 2021$ 


$$
\begin{aligned}
& =-\left\{\sum _ { n = - \infty } ^ { \infty } \left(\int_{0}^{x} z_{n}\left[f_{1}(t) \cos \left(\lambda_{n} t\right)+f_{2}(t) \sin \left(\lambda_{n} t\right)\right] d t \int_{0}^{x} \cos \left(\lambda_{n} t\right) \overline{f_{1}(t)} d t\right.\right. \\
& \left.+\int_{0}^{x}\left[f_{1}(t) \cos \left(\lambda_{n} t\right)+f_{2}(t) \sin \left(\lambda_{n} t\right)\right] d t \int_{0}^{x} \sin \left(\lambda_{n} t\right) \overline{f_{2}(t)} d t\right) \\
& +\frac{1}{\pi} \sum_{n=-\infty}^{\infty}\left(\int_{0}^{x}\left[f_{1}(t) \cos ((n-1 / 2) t)+f_{2}(t) \sin ((n-1 / 2) t)\right] d t \int_{0}^{x} \cos ((n-1 / 2) t) \overline{f_{1}(t)} d t\right. \\
& \left.\left.+\int_{0}^{x}\left[f_{1}(t) \cos (n t)+f_{2}(t) \sin ((n-1 / 2) t)\right] d t \int_{0}^{x} \sin ((n-1 / 2) t) \overline{f_{2}(t)} d t\right)\right\} \\
& =-\left\{\sum_{n=-\infty}^{\infty} z_{n} \int_{0}^{x}\left[f_{1}(t) \cos \left(\lambda_{n} t\right)+f_{2}(t) \sin \left(\lambda_{n} t\right)\right] d t \int_{0}^{x}\left[\overline{f_{1}(t)} \cos \left(\lambda_{n} t\right)+\overline{f_{2}(t)} \sin \left(\lambda_{n} t\right)\right] d t\right. \\
& +\sum_{n=-\infty}^{\infty} \frac{1}{\pi} \int_{0}^{x}\left[f_{1}(t) \cos ((n-1 / 2) t)+f_{2}(t) \sin ((n-1 / 2) t)\right] d t \\
& \left.\times \int_{0}^{x}\left[\overline{f_{1}(t)} \cos ((n-1 / 2) t)+\overline{f_{2}(t)} \sin ((n-1 / 2) t)\right] d t\right\} \\
& =-\sum_{n=-\infty}^{\infty} z_{n}\left|\int_{0}^{x}\left\langle f(t), Y_{0}\left(t, \lambda_{n}\right)\right\rangle d t\right|^{2}-\sum_{n=-\infty}^{\infty} \frac{1}{\pi}\left|\int_{0}^{x}\left\langle f(t), Y_{0}(t,(n-1 / 2))\right\rangle d t\right|^{2}
\end{aligned}
$$

In view of Parseval's identity, we obtain

$$
\|f\|_{L_{2,2}(0, x)}^{2}=\sum_{n=-\infty}^{\infty} \frac{1}{\pi}\left|\int_{0}^{x}\left\langle f(t), Y_{0}(t,(n-1 / 2))\right\rangle d t\right|^{2}
$$

therefore,

$$
\sum_{n=-\infty}^{\infty} z_{n}\left|\int_{0}^{x}\left\langle f(t), Y_{0}\left(t, \lambda_{n}\right)\right\rangle d t\right|^{2}=0
$$

Since $\operatorname{Re} z_{n}<0$ for each $n$, Eq. (22) implies that $\int_{0}^{x}\left\langle f(t), Y_{0}\left(t, \lambda_{n}\right)\right\rangle d t=0$. The latter and the completeness of the system of vectors $\left\{Y_{0}\left(t, \lambda_{n}\right)\right\}$ in $L_{2,2}(0, \pi)$ imply the identity $f(t) \equiv 0$. The unique solvability of Eq. (21) implies [4] that the functions $c(\lambda)$ and $-s(\lambda)$ are the entries of the first row of the monodromy matrix

$$
\tilde{U}(\pi, \lambda)=\left(\begin{array}{cc}
\tilde{c}_{1}(\pi, \lambda) & -\tilde{s}_{2}(\pi, \lambda) \\
\tilde{s}_{1}(\pi, \lambda) & \tilde{c}_{2}(\pi, \lambda)
\end{array}\right)
$$

of problem (1), (2) with the matrix $A$ defined in (10) and some potential $\tilde{V} \in L_{2}(0, \pi)$; i.e.,

$$
c(\lambda)=\tilde{c}_{1}(\pi, \lambda), \quad s(\lambda)=\tilde{s}_{2}(\pi, \lambda) .
$$

By virtue of $(4)$, the characteristic determinant $\tilde{\Delta}(\lambda)$ of this problem has the form

$$
\tilde{\Delta}(\lambda)=\tilde{s}_{1}(\pi, \lambda)-\tilde{s}_{2}(\pi, \lambda)=\tilde{f}(\lambda)
$$


where $\tilde{f} \in P W_{\pi}$. Relations (3), (18), and (23) imply the equality

$$
\tilde{\Delta}\left(\lambda_{n}\right)=\tilde{s}_{1}\left(\pi, \lambda_{n}\right)-\tilde{s}_{2}\left(\pi, \lambda_{n}\right)=\frac{1}{\tilde{s}_{2}\left(\pi, \lambda_{n}\right)}-\tilde{s}_{2}\left(\pi, \lambda_{n}\right)=\frac{1}{s\left(\lambda_{n}\right)}-s\left(\lambda_{n}\right)=f\left(\lambda_{n}\right) .
$$

It follows from the last equality that the function

$$
\Phi(\lambda)=\frac{f(\lambda)-\tilde{\Delta}(\lambda)}{c(\lambda)}=\frac{f(\lambda)-\tilde{f}(\lambda)}{c(\lambda)}
$$

is entire. Since

$$
|f(\lambda)-\tilde{f}(\lambda)|<c_{1} e^{\pi|\operatorname{Im} \lambda|}, \quad c_{1}=\text { const }
$$

we conclude in view of inequality (13) that $|\Phi(\lambda)| \leq c_{2}=$ const if $|\operatorname{Im} \lambda| \geq M$.

Let $H$ stand for the union of vertical segments $\{z:|\operatorname{Re} z|=n,|\operatorname{Im} z| \leq M\}$, where $|n|=N_{0}+1, N_{0}+2, \ldots$ Since the function $c(\lambda)$ is a sine-type function [9], we have $|c(\lambda)|>\delta>0$ for $\lambda \in H$. The last inequality, the estimate (24), and the maximum principle imply the inequality $|\Phi(\lambda)|<c_{3}=$ const in the strip $|\operatorname{Im} \lambda| \leq M$. Consequently, the function $\Phi(\lambda)$ is bounded in the entire complex plane and is constant by Liouville's theorem. Let $|\operatorname{Im} \lambda|=M$. Then, in view of relation (11), we have $\lim _{|\lambda| \rightarrow \infty}(f(\lambda)-\tilde{f}(\lambda))=0$; therefore, $\Phi(\lambda) \equiv 0$, and hence $f(\lambda) \equiv \tilde{\Delta}(\lambda)$. The proof of the theorem is complete.

Examples of functions in the class $P W_{\pi}$ with roots of arbitrarily high multiplicity are known in the literature (see, e.g., $[10,11])$. Note that the existence of one-dimensional boundary value problems with an unboundedly increasing multiplicity of eigenvalues was previously established for the Sturm-Liouville operator and an ordinary differential operator of any even order [10-12].

\section{OPEN ACCESS}

This article is licensed under a Creative Commons Attribution 4.0 International License, which permits use, sharing, adaptation, distribution and reproduction in any medium or format, as long as you give appropriate credit to the original author(s) and the source, provide a link to the Creative Commons license, and indicate if changes were made. The images or other third party material in this article are included in the article's Creative Commons license, unless indicated otherwise in a credit line to the material. If material is not included in the article's Creative Commons license and your intended use is not permitted by statutory regulation or exceeds the permitted use, you will need to obtain permission directly from the copyright holder. To view a copy of this license, visit http://creativecommons.org/licenses/by/4.0/.

\section{REFERENCES}

1. Lunyov, A. and Malamud, M., On the Riesz basis property of root vectors system for $2 \times 2$ Dirac type operators, J. Math. Anal. Appl., 2016, vol. 441, no. 1, pp. 57-103.

2. Kal'menov, T.Sh. and Shaldanbaev, A.Sh., On the structure of the spectrum of Sturm-Liouville boundary value problem on a finite time interval, Izv. Akad. Nauk Resp. Kaz. Ser. Fiz.-Mat., 2000, no. 3, pp. 29-34.

3. Marchenko, V.A., Operatory Shturma-Liuvillya i ikh prilozheniya (Sturm-Liouville Operators and Their Applications), Kiev: Nauk. Dumka, 1977.

4. Tkachenko, V., Non-self-adjoint periodic Dirac operators, Oper. Theory Adv. Appl., 2001, vol. 123, pp. $485-512$.

5. Misyura, T.V., Characteristic of the spectra of periodic and antiperiodic boundary value problems generated by the Dirac operation. II, Teor. Funkts. Funkts. Anal. Ikh Pril., 1979, vol. 31, pp. 102-109.

6. Tkachenko, V., Non-self-adjoint periodic Dirac operators with finite-band spectra, Int. Equat. Oper. Theory, 2000, vol. 36, pp. 325-348.

7. Levin, B.Ya., Tselye funktsii (kurs lektsii) (Entire Functions (a Course of Lectures)), Moscow: Mosk. Gos. Univ., 1971. 
8. Sedletskii, A.M., Nonharmonic analysis, Itogi Nauki Tekh. Ser. Sovrem. Mat. Ee Pril. Temat. Obz., 2006, vol. 96, pp. 106-211.

9. Levin, B.Ya. and Ostrovskii, I.V., On small disturbances of the set of roots of sine-type functions, Izv. Akad. Nauk SSSR. Ser. Mat., 1979, vol. 43, no. 1, pp. 87-110.

10. Makin, A.S., On a two-point boundary value problem for the Sturm-Liouville operator with a nonclassical asymptotics of the spectrum, Differ. Equations, 2013, vol. 49, no. 5, pp. 536-544.

11. Makin, A.S., Problem with nonclassical eigenvalue asymptotics, Differ. Equations, 2015, vol. 51, no. 3, pp. 318-324.

12. Makin, A., Two-point boundary value problems with nonclassical asymptotics on the spectrum, Electron. J. Differ. Equat., 2018, vol. 2018, no. 95, pp. 1-7. 